me to be a case of special pleading.

The Lopsided Ape is nevertheless an excellent read, enlivened by many anecdotes, historical details and jokes. On the problems of the speech-deprived right hemisphere, Corballis (a New Zealander) remarks in a footnote that the disadvantages are well understood by his fellow countrymen "who also share a hemisphere with a more talkative neighbour".

Michael Morgan is in the Department of Pharmacology, University of Edinburgh, 1 George Square, Edinburgh EH8 9JZ, UK.

\section{Birth of a new frontier}

David J. Miller

The Meson Factories. Edited by Torleif E. O. Ericson, Vernon W. Hughes and Darragh E. Nagle. University of California Press: 1992. Pp. 870. \$75.

THE obvious frontiers of knowledge are not the only places where new scientific disciplines grow. In the period covered by this substantial volume of reprints and commentaries (the mid-1970s to the late 1980s), the high-energy frontier of particle physics had already reached hundreds of gigaelectronvolts, but the impressively diverse work described here is all at or below a few hundred megaelectronvolts, based particularly on the programmes of three meson factories, a proton linear accelerator at Los Alamos (LAMPF), an isochronous ring at Zurich (formerly SIN, now PSI) and an $\mathrm{H}^{-}$ cyclotron in Vancouver (TRIUMF). These factories are distinguished from the ubiquitous cyclotrons of the $1950 \mathrm{~s}$ and 60 s by their high beam currents (0.1-1 millamperes), which have made whole new classes of study possible.

The 50-page introduction gives the context in which the machines were built. Although the editors pick out their own list of outstanding results, many readers will find equally valid alternative lists from their own browsing through the reprints (121 in all), which cover the machines themselves (9), particle physics (27), nuclear physics (35), atomic physics (26), condensed matter (18) and applications (6).

In the section devoted to particle physics we are reminded that the standard model of electroweak theory has been tested as rigorously in muon ( $\mu$-meson) and pion ( $\pi$-meson) decays as it has in neutrino scattering or $\mathrm{Z}^{0}$ and $\mathrm{W}^{ \pm}$particle physics. There are classic papers on the mean lifetime of the positive muon (the baseline for all fits to the electroweak parameters) and on the limits to

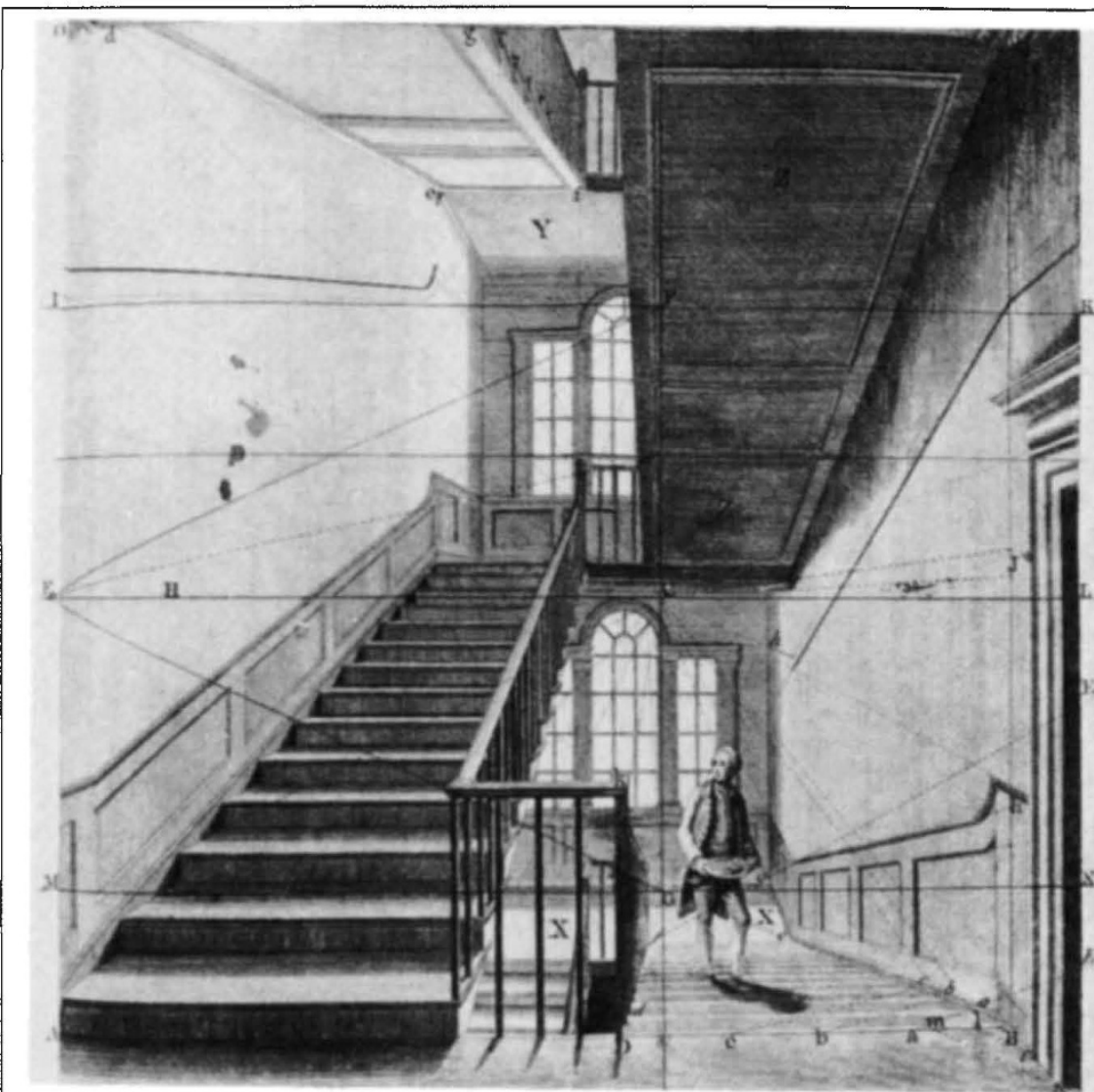

Perspectival study of a staircase, from Malton's A Complete Treatise on Perspective (1779). The plate is taken from Martin Kemp's beautifully produced book The Science of Art: Optical Themes in Western Art from Brunelleschi to Seurat, now published in paperback by Yale University Press, price $\$ 35, £ 19.95$.

forbidden decays of muons, for example to an electron and a photon. Highprecision pion-nucleon and nucleonnucleon scattering experiments measure the low-energy scattering amplitudes and establish the degree of charge-symmetry breaking.

There are two papers on chargesymmetry breaking among the nuclearphysics reprints, and much detailed work on spin-dependent scattering amplitudes is described. Pion double-charge exchange is covered by six reprints.

It is in the atomic-physics section that totally new disciplines emerge most clearly. Muonium (an artificial electronmuon 'atom') provides a new laboratory for the precise study of quantum electrodynamics, without the uncertainties caused by the structure of the proton. There are reports of muonium's production into vacuum and the measurement of its hyperfine splittings. Its Lamb shift gives a new precise value for the fine structure constant and for testing model calculations. Muonic atoms (in which a Muon replaces an electron) are another new laboratory. The muon's small orbit in heavy atoms means that vacuum polarization effects can be tested more rigorously than in any other system.

Perhaps the most important application of low-energy meson beams will turn out to be muon-catalysed fusion of deuterium and tritium. The volume includes the theoretical paper by Gerstein and Ponomarev showing that resonant formation of an excited muonic molecule should allow each negative muon to catalyse roughly 100 fusions; also reprinted are the first experimental results indicating this level of catalysis.

Many of the reprints on condensed matter concern applications of the muon spin-rotation technique, although there is also a paper on the use of the LAMPF pulsed thermal neutron facility to study a high-temperature superconductor. Applications are covered by five reprints on medical physics, and one on radiation damage in aluminium.

Students starting $\mathrm{PhD}$ studies in any of the fields covered - whether at a meson factory, at ISIS, at Grenoble or elsewhere - should be required to read the whole volume to see the context in which they will be working. But it is a good browse for any physicist, and something to put on the library purchase list. (It has the advantage of being too heavy to be easily stolen.)

David J. Miller is in the Department of Physics and Astronomy, University College London, Gower Street, London WC1E 6BT, UK. 